\title{
ON PRODUCTS OF POLYNOMIALS
}

\author{
D. W. MASSER AND J. WOLBERT
}

(Communicated by William Adams)

\begin{abstract}
For a complex polynomial $P$ in a single variable, let $H(P)$ be the maximum of the absolute values of its coefficients. Given nonnegative integers $n_{1}$ and $n_{2}$, it is well known that $\mu\left(n_{1}, n_{2}\right)=\inf H\left(P_{1} P_{2}\right) /\left(H\left(P_{1}\right) H\left(P_{2}\right)\right)>0$, where the infimum is taken over all such polynomials $P_{1}$ and $P_{2}$ of degrees $n_{1}$ and $n_{2}$ respectively. We determine here the exact values of $\mu(1, n)$ for every $n$.
\end{abstract}

For a complex polynomial $P$ in a single variable $x$ let $H(P)$ denote the height of $P$; that is, the maximum of the absolute values of its coefficients. For nonnegative integers $n_{1}$ and $n_{2}$ we define

$$
\mu\left(n_{1}, n_{2}\right)=\inf H\left(P_{1} P_{2}\right) /\left(H\left(P_{1}\right) H\left(P_{2}\right)\right),
$$

where the infimum is taken over all polynomials $P_{1}$ and $P_{2}$ of degrees $n_{1}$ and $n_{2}$ respectively. It is easy to see, for example by compactness arguments, that $\mu\left(n_{1}, n_{2}\right)>0$, and a well-known result of Gelfond [G, p. 139] gives the estimate

$$
\mu\left(n_{1}, n_{2}\right) \geq 2^{-\left(n_{1}+n_{2}\right)}\left\{2 /\left(1+n_{1}+n_{2}\right)\right\} .
$$

The example $P_{1}=(x-1)^{n}, P_{2}=(x+1)^{n}$ shows that $\mu(n, n) \leq 2^{-n}(n+1)$, and therefore the exponential dependence cannot be avoided in general; see also the more elaborate examples constructed by Gelfond himself in [G, p. 140].

We are concerned here with the exact values of $\mu\left(n_{1}, n_{2}\right)$. It is trivial that $\mu(0, n)=1$, and it is just an exercise (see, for example, [S]) to prove that $\mu(1,1)=\frac{1}{2}(\sqrt{5}-1)$. Apart from this, not much seems to be known. In the present note we evaluate $\mu(1, n)$ in simple finite form for all $n$. It turns out that this does not depend exponentially on $n$.

Our result is as follows.

Theorem. For a nonnegative integer $n$ let $m$ be the greatest integer not exceeding $\frac{1}{2}(n+1)$. Then

$$
(\mu(1, n))^{-1}=1+\tau+\cdots+\tau^{m},
$$

where $\tau$ is the unique positive real number satisfying

$$
1+\tau+\cdots+\tau^{m}=\tau^{-1}+\tau^{-2}+\cdots+\tau^{-(n-m+1)} .
$$

Received by the editors December 19, 1990.

1980 Mathematics Subject Classification (1985 Revision). Primary 12D99. 
In particular, if $n$ is even we have $\tau=1$, and so $\mu(1, n)=2 /(n+2)$. If $n$ is odd it can be shown that $\mu(1, n)<2 /(n+2)$, but that the difference is at most of order $n^{-3}$ for large $n$.

The proof of the Theorem will occupy several lemmas. From now on we write $\mu=\mu(1, n)$, and in general we suppress the dependence on $n$ in our notation. For real $t>0$ and any integer $k$ with $0 \leq k \leq n$ we define

$$
A_{k}(t)=1+t+\cdots+t^{k}, \quad B_{k}(t)=t^{-1}+t^{-2}+\cdots+t^{-(n-k+1)}
$$

and

We further define

$$
C_{k}(t)=\min \left(A_{k}(t), B_{k}(t)\right)
$$

$$
\nu(t)=\max _{0 \leq k \leq n} C_{k}(t), \quad \nu=\sup _{0<t \leq 1} \nu(t) .
$$

Lemma 1. We have $\mu^{-1} \leq \nu$.

Proof. Fix a complex number $z$ with $0<|z| \leq 1$, and let $L$ denote the polynomial $L(x)=x+z$. Let also $P=P(x)=c_{0} x^{n}+\cdots+c_{n}$ be an arbitrary polynomial of degree $n$, and suppose the product $L P$ has height $H$. Then we have the inequalities

$$
\left|c_{0}\right| \leq H,\left|c_{1}+z c_{0}\right| \leq H, \ldots,\left|c_{n}+z c_{n-1}\right| \leq H,\left|z c_{n}\right| \leq H .
$$

Solving these from left to right, we find that

$$
\left|c_{k}\right| \leq H A_{k}(t) \quad(0 \leq k \leq n),
$$

where $t=|z|$. But solving from right to left, we get

$$
\left|c_{k}\right| \leq H B_{k}(t) \quad(0 \leq k \leq n) \text {. }
$$

Therefore

$$
\left|c_{k}\right| \leq H C_{k}(t) \quad(0 \leq k \leq n)
$$

and so $H(P) \leq H \nu(t) \leq H \nu$. Since $H(L)=1$, we have shown that

$$
H(L P) /(H(L) H(P)) \geq \nu^{-1}
$$

for any $L=x+z$ with $0<|z| \leq 1$.

The inequality ( 1 ) is readily extended to all $L$ of degree 1 . First, it holds for $L=x$ by continuity. Next, by considering reciprocal polynomials we deduce it for $L=z x+1$ and any $z$ with $0<|z| \leq 1$. Finally it follows for arbitrary $L$ by homogeneity. The resulting extended inequality (1) says that $\mu \geq \nu^{-1}$ or $\mu^{-1} \leq \nu$ as desired. This proves the present lemma.

Lemma 2. Let $k$ be an integer with $0 \leq k \leq n$. Then the equation $A_{k}(t)=B_{k}(t)$ has a unique positive real root $t=t_{k}$. Further if $n=2 m$ is even we have

$$
0<t_{n}<t_{n-1}<\cdots<t_{m}=1<t_{m-1}<\cdots<t_{0},
$$

while if $n=2 m-1$ is odd then

$$
0<t_{n}<t_{n-1}<\cdots<t_{m}<1<t_{m-1}<\cdots<t_{0} .
$$

Proof. The existence of $t_{k}$ is clear, for example, from Descartes Rule of Signs. The same rule also gives uniqueness, or we could simply appeal to the fact, used often in this note, that $A_{k}(t)-B_{k}(t)$ is increasing in $t$. Also

$$
0=t_{k}\left(A_{k}\left(t_{k}\right)-B_{k}\left(t_{k}\right)\right)=B_{n-k}\left(t_{k}^{-1}\right)-A_{n-k}\left(t_{k}^{-1}\right) \text {, }
$$


from which it follows that $t_{n-k}=t_{k}^{-1}$. Taking $k=m$ we find that $t_{m}=1$ for even $n$ and $t_{m} t_{m-1}=1$ for odd $n$. But also

$$
0=A_{k}\left(t_{k}\right)-B_{k}\left(t_{k}\right)<A_{k+1}\left(t_{k}\right)-B_{k+1}\left(t_{k}\right)
$$

for $k<n$, which shows that $t_{k+1}<t_{k}$. These observations together imply the inequalities of the present lemma.

Lemma 3. Defining $\alpha_{k}=A_{k}\left(t_{k}\right)(0 \leq k \leq n)$ we have $\nu=\max _{m \leq k \leq n} \alpha_{k}$.

Proof. We consider even $n=2 m$ first. The interval $I=(0,1]$ then breaks into the intervals

$$
I_{n}=\left(0, t_{n}\right], I_{n-1}=\left[t_{n}, t_{n-1}\right], \ldots, I_{m}=\left[t_{m+1}, t_{m}\right] .
$$

Therefore

$$
\nu=\sup _{t \in I} \nu(t)=\max _{m \leq h \leq n} \nu_{h}
$$

where

$$
\nu_{h}=\sup _{t \in I_{h}} \nu(t)=\sup _{t \in I_{h}} \max _{0 \leq k \leq n} C_{k}(t) .
$$

Now fix $h$ with $m \leq h<n$, and also $t$ in $I_{h}$. For any $k$ with $0 \leq k \leq h$ we have $t \leq t_{h} \leq t_{k}$ and consequently $A_{k}(t) \leq B_{k}(t)$. So

$$
C_{k}(t)=A_{k}(t) \quad(0 \leq k \leq h) .
$$

On the other hand, for any $k$ with $h+1 \leq k \leq n$ we have $t \geq t_{h+1} \geq t_{k}$, so

$$
C_{k}(t)=B_{k}(t) \quad(h+1 \leq k \leq n) .
$$

Thus

$$
\begin{aligned}
\nu_{h} & =\sup _{t \in I_{h}} \max \left\{A_{0}(t), \ldots, A_{h}(t), B_{h+1}(t), \ldots, B_{n}(t)\right\} \\
& =\sup _{t \in I_{h}} \max \left(A_{h}(t), B_{h+1}(t)\right) .
\end{aligned}
$$

But since $A_{h}$ is increasing, $B_{h+1}$ is decreasing, and $I_{h}=\left[t_{h+1}, t_{h}\right]$, it is easy to see that this last expression is $\max \left(A_{h}\left(t_{h}\right), B_{h+1}\left(t_{h+1}\right)\right)$. Therefore

$$
\nu_{h}=\max \left(\alpha_{h}, \alpha_{h+1}\right) \quad(m \leq h<n) .
$$

The same arguments with $h=n$ give

$$
\nu_{n}=\alpha_{n}
$$

without extra difficulty.

Now (2), (4), and (5) establish the present lemma when $n$ is even.

The proof for odd $n=2 m-1$ is similar. Then $I$ splits into $I_{n}, \ldots, I_{m}$ as above together with $I_{m-1}=\left[t_{m}, 1\right]$, and so

$$
\nu=\max _{m-1 \leq h \leq n} \nu_{h}
$$

with $\nu_{h}$ still defined by (3). If $m \leq h \leq n$ we obtain (4) and (5) without changes. For $h=m-1, t$ in $I_{m-1}$, and $0 \leq k \leq m-1$ we have $t \leq 1<t_{k}$, and so

$$
C_{k}(t)=A_{k}(t) \quad(0 \leq k \leq m-1)
$$


while for $m \leq k \leq n$ we have $t \geq t_{m} \geq t_{k}$ and so

$$
C_{k}(t)=B_{k}(t) \quad(m \leq k \leq n) .
$$

Thus

$$
\begin{aligned}
\nu_{m-1} & =\sup _{t \in I_{m-1}} \max \left\{A_{0}(t), \ldots, A_{m-1}(t), B_{m}(t), \ldots, B_{n}(t)\right\} \\
& =\sup _{t \in I_{m-1}} \max \left(A_{m-1}(t), B_{m}(t)\right)=\max \left(A_{m-1}(1), B_{m}\left(t_{m}\right)\right) .
\end{aligned}
$$

Now $\alpha_{m}=B_{m}\left(t_{m}\right)>B_{m}(1)=A_{m-1}(1)$ so

$$
\nu_{m-1}=\alpha_{m} \text {. }
$$

Finally (6), (4), (5), and (7) establish the present lemma when $n$ is odd, and this completes the proof.

Lemma 4. We have $\mu^{-1} \geq \nu$.

Proof. By Lemma 3, there exists $k$ with $m \leq k \leq n, \nu=\alpha_{k}$. Consider the polynomial

$$
Q=Q(x)=x^{n-k+1}\left(A_{k}(x)-B_{k}(x)\right) .
$$

Since $Q\left(t_{k}\right)=0$, we can write $Q=L P$ for $L=L(x)=x-t_{k}$ and some polynomial $P=P(x)$. Clearly $H(L) \geq 1$ and $H(L P)=1$. Also the coefficient of $x^{n-k}$ in

$$
\begin{aligned}
& P(x)=Q(x) /\left(x-t_{k}\right) \\
& \quad=-x^{-1}\left(1+t_{k} x^{-1}+t_{k}^{2} x^{-2}+\cdots\right)\left(x^{n+1}+\cdots+x^{n-k+1}-x^{n-k}-\cdots-x-1\right)
\end{aligned}
$$

is

$$
-\left(1+t_{k}+\cdots+t_{k}^{k}\right)=-\alpha_{k}
$$

Thus $H(P) \geq \alpha_{k}$, and

$$
H(L P) /(H(L) H(P)) \leq \alpha_{k}^{-1}=\nu^{-1},
$$

so $\mu \leq \nu^{-1}$ or $\mu^{-1} \geq \nu$ as desired. This completes the proof.

At this stage we can combine Lemmas 1,3 , and 4 to conclude that

$$
\mu^{-1}=\max _{m \leq k \leq n} \alpha_{k}
$$

Thus for any fixed $n$ the determination of $\mu=\mu(1, n)$ is a matter of finite computation. The first few values are

$$
\mu(1,0)=1, \quad \mu(1,1)=\frac{1}{2}(\sqrt{5}-1), \quad \mu(1,2)=\frac{1}{2},
$$

$\mu(1,3)=0.38939 \ldots$ the smallest positive real root of $t^{4}+t^{3}-2 t^{2}-2 t+1=0$, and $\mu(1,4)=\frac{1}{3}$.

To prove the Theorem we must show that the right-hand side of (8) is $\alpha_{m}$. This requires some rather tedious calculations.

Lemma 5. For an integer $k$ with $0 \leq k \leq n$ and real $t$ with $0<t \leq 1$ we have

$$
A_{k}(t)=a_{k}(t)+\mathscr{A}_{k}(t), \quad B_{k}(t)=b_{k}(t)+\mathscr{B}_{k}(t),
$$

where

$$
a_{k}(t)=k+1+\frac{1}{2} k(k+1)(t-1)+\frac{1}{6} k(k+1)(k-1)(t-1)^{2},
$$




$$
\begin{aligned}
b_{k}(t)= & n-k+1-\frac{1}{2}(n-k+1)(n-k+2)(t-1) \\
& +\frac{1}{6}(n-k+1)(n-k+2)(n-k+3)(t-1)^{2},
\end{aligned}
$$

and

$$
\left|\mathscr{A}_{k}(t)\right| \leq \frac{1}{24} k^{4}|t-1|^{3}, \quad\left|\mathscr{B}_{k}(t)\right| \leq \frac{1}{24} t^{-(n-k+4)}(n-k+3)^{4}|t-1|^{3} .
$$

Proof. Clearly $a_{k}(t)$ consists of the first three terms in the Taylor expansion of

$$
A_{k}(t)=\left\{(1+s)^{k+1}-1\right\} / s \quad(s=t-1)
$$

about $t=1$. It follows that

$$
\mathscr{A}_{k}(t)=\frac{1}{6} A_{k}^{\prime \prime \prime}(\tau)(t-1)^{3}
$$

for some $\tau$ between $t$ and 1 . Also

$$
\left|A_{k}^{\prime \prime \prime}(\tau)\right| \leq A_{k}^{\prime \prime \prime}(1)=\frac{1}{4} k(k+1)(k-1)(k-2) \leq \frac{1}{4} k^{4},
$$

which yields the required estimate for $\mathscr{A}_{k}(t)$.

The proof for

$$
B_{k}(t)=\left\{1-(1+s)^{-(n-k+1)}\right\} / s \quad(s=t-1)
$$

is similar, except that now

$$
\left|B_{k}^{\prime \prime \prime}(\tau)\right| \leq \tau^{-(n-k+4)}\left|B_{k}^{\prime \prime \prime}(1)\right| \leq t^{-(n-k+4)}\left|B_{k}^{\prime \prime \prime}(1)\right|
$$

and

$$
B_{k}^{\prime \prime \prime}(1)=-\frac{1}{4}(n-k+1)(n-k+2)(n-k+3)(n-k+4) .
$$

The present lemma is thus established.

Lemma 6. If $n \geq 2$ then $\alpha_{m+1}<\alpha_{m}$.

Proof. First assume $n=2 m$ is even. Suppose we can find a real number $w>0$ such that

$$
m+1>A_{m+1}(w)>B_{m+1}(w) .
$$

Then $t_{m+1}<w$, so that

$$
\alpha_{m+1}=A_{m+1}\left(t_{m+1}\right)<A_{m+1}(w)<m+1=\alpha_{m}
$$

and our present lemma follows.

For $m=1,2, \ldots, 9$ we verify (9) by direct calculation, the values of $w$ being $0.6,0.8,0.88,0.925,0.945,0.96,0.97,0.976,0.9805$ respectively. For $m \geq 10$ we will show that (9) holds with

$$
w=1-2 m^{-2}+4 m^{-3} \text {. }
$$

This can also be checked directly for $m \leq 19$, so we shall henceforth suppose $m \geq 20$.

First, we find that

$$
a_{m+1}(w)=m+1-\frac{1}{3} m^{-1}+\frac{10}{3} m^{-2}+\frac{8}{3} m^{-4}+\frac{16}{3} m^{-5}
$$

and

$$
\left|\mathscr{A}_{m+1}(w)\right| \leq \frac{1}{24}(m+1)^{4}\left(2 m^{-2}\right)^{3}<m^{-2} .
$$

It follows easily that

$$
A_{m+1}(w)=a_{m+1}(w)+\mathscr{A}_{m+1}(w)<m+1,
$$


and also

$$
A_{m+1}(w)>m+1-\frac{1}{3} m^{-1}
$$

Next, we have

$$
b_{m+1}(w)=m+1-\frac{1}{3} m^{-1}-\frac{8}{3} m^{-2}-4 m^{-3}+\frac{8}{3} m^{-4}+\frac{16}{3} m^{-5}
$$

and

$$
\left|\mathscr{B}_{m+1}(w)\right| \leq \frac{1}{24} w^{-(m+3)}(m+2)^{4}\left(2 m^{-2}\right)^{3}<m^{-2}
$$

Thus

$$
B_{m+1}(w)=b_{m+1}(w)+\mathscr{B}_{m+1}(w)<m+1-\frac{1}{3} m^{-1} \text {. }
$$

Comparing this with (11) and recalling (10), we see that the present lemma is proved for even $n$.

For odd $n=2 m-1$ we prove it in a similar fashion by exhibiting positive real $u, v$ with

$$
B_{m}(u)>A_{m}(u)>A_{m+1}(v)>B_{m+1}(v) .
$$

In fact the choices $(u, v)=(0.8,0.6)$ and $(0.9,0.8)$ work for $m=2$ and 3 respectively, while the choice

$$
u=1-m^{-2}, \quad v=1-3 m^{-2}+4 m^{-3}
$$

works for all $m \geq 4$. We find that, up to terms of order $m^{-2}$, the expressions in (12) are $m+\frac{1}{2}+\frac{2}{3} m^{-1}, m+\frac{1}{2}-\frac{1}{3} m^{-1}, m+\frac{1}{2}-m^{-1}, m+1-2 m^{-1}$ respectively, so the inequalities in (12) are somewhat looser than those in (9). The details of the calculation can safely be left to the reader and his computer, and so we will regard the present lemma as completely proved.

Lemma 7. For any integer $k$ with $m \leq k \leq n$ we have $\alpha_{k} \leq \alpha_{m}$.

Proof. If $n \leq 3$ then $m \leq k \leq n$ implies $k=m$ or $m+1$, so the result follows from Lemma 6 . Thus we can suppose $n \geq 4$. Now when $t_{k} \neq 1$ we have

$$
\alpha_{k}=\left(1-\tau^{k+1}\right) /(1-\tau)
$$

where $\tau=t_{k}$ satisfies

$$
\tau^{n+2}-2 \tau^{n-k+1}+1=0 .
$$

Calculating $\tau^{k}$ from (14) and substituting it into (13), we find that

$$
\alpha_{k}=f\left(t_{k}\right),
$$

where $f(t)$ is the continuous function for $t>0$ defined by

$$
f(t)=\left(1-t^{n+2}\right) /\left\{(1-t)\left(1+t^{n+2}\right)\right\} \text {. }
$$

Also (15) can be checked directly when $t_{k}=1$. If the present lemma is false, then there exists $k$ with $m \leq k \leq n$ and $\alpha_{k}>\alpha_{m}$. By Lemma 6, we have $k \geq m+2$, and the inequalities $\alpha_{k}>\alpha_{m+1}<\alpha_{m}$ read

$$
f\left(t_{k}\right)>f\left(t_{m+1}\right)<f\left(t_{m}\right) .
$$

Since $0<t_{k}<t_{m+1}<t_{m} \leq 1$, this implies that the function $f(t)$ has a local minimum at some $\tau^{\prime}$ with $0<\tau^{\prime}<1$. Now it is readily verified by differentiation that the critical points of $f(t)$ for $t>0$ satisfy

$$
t^{2 n+4}-(2 n+4) t^{n+2}+(2 n+4) t^{n+1}-1=0 \text {. }
$$


This equation has a double root at $t=1$, and Descartes Rule shows that there is exactly one more positive root; this must therefore be $\tau^{\prime}$. Also $f^{\prime}(1)=$ $-\frac{1}{4}(n+2) \neq 0$, and so the local minimum $\tau^{\prime}$ is the only critical point of $f(t)$ for $t>0$. But since $f(t)>0$ for $t>0$ and $f(t) \rightarrow 0$ as $t \rightarrow \infty$, this is not possible. This contradiction proves the present lemma.

Our Theorem is now an immediate consequence of (8) and Lemma 7.

\section{REFERENCES}

[G] A. Gelfond, Transcendental and algebraic numbers, Dover, New York, 1960.

[S] Solution to problem E2217, Amer. Math. Monthly 78 (1971), 79.

Department of Mathematics, University of Michigan, Ann Arbor, Michigan 481091003

Current address, D. W. Masser: Mathematisches Institut, Universität Basel, Rheinsprung 21, 4056 Basel, Switzerland

Current address, J. Wolbert: Department of Mathematics, University of Chicago, 5734 S. University Ave., Chicago, Illinois 60637 